\title{
Culture result of smear-positive sputum samples after 2 months of antituberculous treatment
}

\section{To the Editors:}

The correlation between sputum smear for acid-fast bacilli (AFB) and culture for Mycobacterium tuberculosis is usually good before antituberculous treatment (ATT), but it is not so good after treatment has started. In previous studies, up to $23.5 \%$ of patients had sputum that remained smear positive after 2 months of ATT [1]. Old age, high bacillary load, presence of pulmonary cavitary lesions, treatment interruption and emergence of resistant strains are risk factors of persistent smear positivity. Determining whether these sputum samples contain viable tuberculosis (TB) bacilli is crucial because it indicates higher rates of relapse [2,3]. Meanwhile, for patients without viable TB bacilli, modifying treatment regimens and isolation appears to be redundant. However, the results of mycobacterial culture are only available after 2 weeks, even when using fluorometric liquid culture technique. Despite having good sensitivity and specificity, commercialised nucleic acid amplification tests are expensive and do not discriminate between viable and dead bacilli and, therefore, are not recommended for TB patients receiving ATT. Thus, we conducted a study to identify clinical factors for predicting the culture results of the persistent smear-positive sputum samples in a 2,100 bed, tertiary-care referral centre in northern Taiwan and an 800 bed local teaching hospital in southern Taiwan.

In the two hospitals, all sputum specimens were processed by adding $\mathrm{NaOH}$-citrate- $\mathrm{N}$-acetyl-L-cysteine at room temperature for $15 \mathrm{~min}$. After centrifugation, the precipitate was resuspended [4]. Before October 2006, smears for AFB of the processed samples were stained using the Kinyoun method. From November 2006, the smear was stained with auraminerhodamine fluorochrome [5]. Smears were graded according to guidelines established by the American Thoracic Society [6] and the highest smear grading was recorded. All collected specimens were sent for mycobacterial culture using two types of culture media: Middlebrook 7H11 selective agar with antimicrobials (Remel, Lexena, KA, USA) and the fluorometric BACTEC technique (BACTEC Mycobacterium Growth Indicator Tube 960 system; Becton-Dickinson, Heidelberg, Germany). Growth on either of the two media was considered as a positive culture. Mycobacterial species were identified using conventional biochemical testing [5]. The susceptibility test of first-line antituberculous drugs was examined using two concentrations of isoniazid $\left(0.2\right.$ and $\left.1.0 \mu \mathrm{g} \cdot \mathrm{mL}^{-1}\right)$ and ethambutol $\left(7.5\right.$ and $\left.15 \mu \mathrm{g} \cdot \mathrm{mL}^{-1}\right)$, and one concentration of rifampicin $\left(1.0 \mu \mathrm{g} \cdot \mathrm{mL}^{-1}\right)$. For isoniazid and ethambutol, low-level resistance was defined as resistance.

Between July 2003 and June 2008, 1,925 patients were diagnosed with pulmonary TB. Among them, 1,620 (84.2\%) patients were culture positive with 898 (46.6\% of total; $55.4 \%$ of culture positive) being smear positive. Among the 898 patients who were smear positive and culture positive, samples were collected after 2 months of ATT in 812 (90.4\%) patients and remained smear positive for AFB in 60 (6.7\%) patients. The 60 patients whose sputum remained smear positive after 2 months of antituberculous treatment collected three earlymorning sputum samples after 2 months of treatment. Among the 60 AFB smear-positive patients, sputum samples were still culture positive for $M$. tuberculosis in 33 (55\%; culture-positive group), including 18 (55\%) patients having three positive cultures, seven $(21 \%)$ having two positive cultures, and the remaining eight $(24 \%)$ having a single positive culture. Sputum samples were culture negative after 2 months of ATT in the remaining 27 (45\%; culture-negative group) patients. All of them were Taiwanese and were not infected by HIV.

All patients in our study cohort started their ATT with a standard regimen consisting of daily isoniazid $\left(5 \mathrm{mg} \cdot \mathrm{kg}^{-1}\right)$, rifampicin $\left(10 \mathrm{mg} \cdot \mathrm{kg}^{-1}\right)$ and ethambutol $\left(15-20 \mathrm{mg} \cdot \mathrm{kg}^{-1}\right)$, plus pyrazinamide (20-25 $\mathrm{mg} \cdot \mathrm{kg}^{-1}$ ) (also known as HREZ) for the initial 2 months (intensive phase), followed by daily isoniazid and rifampicin for another 4 months (continuation phase) [7]. Treatment interruption in the first 2 months was defined as administration of $<56$ doses of the drug within 8 weeks. The treatment outcomes were recorded according to the definition recommended by the World Health Organization [8]. Meanwhile, directly observed therapy was implemented by the Taiwan government, which covered the entire course of ATT from April 2006. All statistical analyses were performed by using SPSS/PC Version 16 (SPSS, Chicago, IL, USA).

The initial clinical characteristics of the two groups were similar (table 1). After 2 months of ATT, significantly more patients in the culture-positive group had high smear grading, no radiographic improvement and incomplete remission of clinical symptoms compared with those in the culture-negative group. Treatment interruption within the first 2 months of ATT was more common in the culture-positive group. The fatality rate was higher in the culture-positive group. Logistic regression analysis revealed that incomplete remission of symptoms (OR 14.47, 95\% CI 2.33-90.04), no radiographic improvement (OR 19.60, 95\% CI 3.49-110.18) and noncontinuous use of isoniazid (OR 19.67, 95\% CI 1.51-256.67) were independently associated with culture positivity.

In the culture-positive group, all had at least one of these factors. For the 41 patients from our study cohort who had only one of these factors, $33(80.5 \%)$ patients belonged to the culture-positive group. Drug susceptibility testing was repeated for the M. tuberculosis isolates collected after 2 months of ATT in $29(88 \%)$ patients. None of these isolates had a different resistant pattern to the pre-treatment isolate.

In our cohort, $24(40 \%)$ patients suffered from adverse effects in the first 2 months of ATT, including cutaneous itchiness in $11(46 \%)$, treatment-related hepatotoxicity (defined according to the established guidelines) [7] in eight (33\%), and two (8\%) patients each for gastrointestinal upset, peripheral neuropathy and blurred vision. The most common reason for treatment 
TABLE 1 Clinical characteristics before and after 2 months of treatment

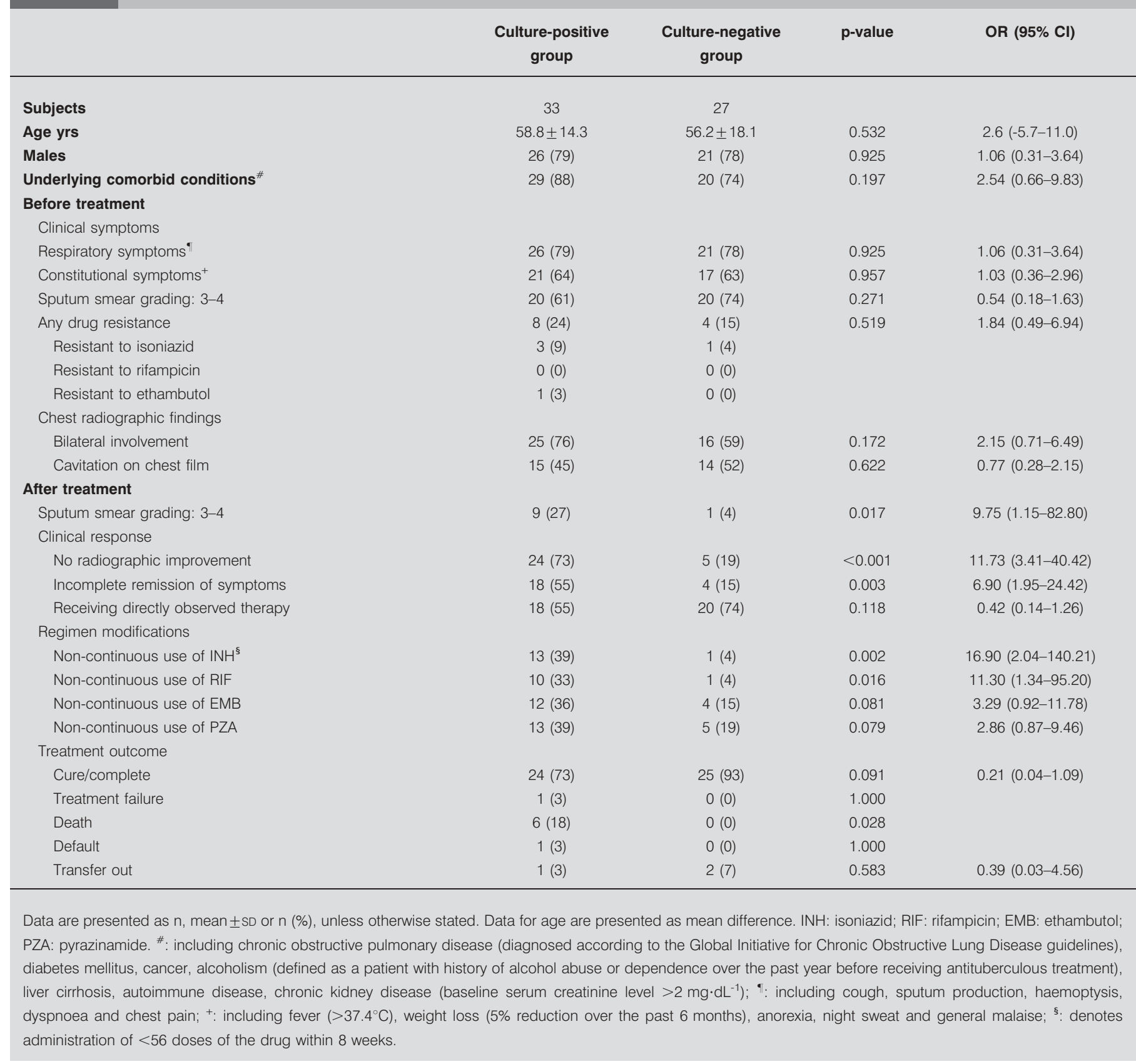

interruption was drug toxicity (24 out of $28 ; 86 \%$ ). Seven (29\%) patients resumed all antituberculous medications after a temporary stop. Eight (33\%) received isoniazid and rifampicin plus ethambutol. Second-line antituberculous drugs were prescribed in nine $(32 \%)$ patients, with fluoroquinolones and streptomycin being most commonly used. EMB was discontinued after knowing the clinical isolate was an all-susceptible strain in three $(11 \%)$ patients. The remaining patient temporarily stopped all medications due to upper gastrointestinal bleeding.

Our findings agree with previous studies showing that INH is the most potent bactericidal agent responsible for killing rapidly growing $\mathrm{TB}$ bacilli in lesions; it is an important sterilising drug for killing the remaining populations of bacilli while used together with certain regimens. Non-continuous use of INH during the intensive phase, when there is the highest bacillary population, places one at risk of failing sterilisation of sputum. It is important to realise that most interruptions to therapy are due to drug toxicity. When a patient's adherence is good, intolerance is the main obstacle for continuing antituberculous medications.

Our study showed that the initial sputum bacillary load and disease extent were not different in the culture-positive and culture-negative groups. These findings imply that with good compliance for medications, even patients with extensive disease could achieve sterile sputum after 2 months of therapy. 
Current guidelines on isolation of patients with suspected infectious pulmonary TB is cautious but probably imperfect [9]. As suggested in our study, smear-positive sputum after 2 months of ATT does not always indicate the presence of viable TB bacilli. Our analyses suggest that among patients whose sputum samples remain smear positive after 2 months of ATT, those who have incomplete remission of symptoms, no radiographic improvement, or who do not continuously receive isoniazid were more likely to have viable TB bacilli in their sputum. When none of these three risk factors appears, the chance of remaining culture positive seemed to be low. If culturable TB bacilli in the sputum were regarded as infective [10], contact protection should not be necessary, and treatment plans should not be changed for these patients. In addition, sputum cultures were heavily positive in only three-quarters of the culture-positive patients. Too much effort on isolation and regimen modification based only on sputum-smear positivity may cause unnecessary anxiety to patients and their families, and excessive expenditure on isolation facilities and overcrowding of isolation rooms, which may even increase the burden of effective TB control. And for the culture-positive patients, whether or not treatment was interrupted, their TB isolates did not acquire drug resistance after 2 months of treatment. This further confirmed that the emergence of a resistant strain may not become a risk factor for culture positivity under combined chemotherapy within this time frame.

In conclusion, of the TB patients who remained smear positive after 2 months of ATT, viable TB bacilli were more likely to be present in those without complete remission of symptoms, radiographic improvement or continuous use of isoniazid. In order to prevent the unnecessary waste of medical resources, contact isolation may not be necessary for those who have none of the three factors.

C-S. Lim*, C-H. Lee", Y-J. Chien ${ }^{\Uparrow}$, J-Y. Wang*, L-N. Lee ${ }^{+}$, C-J. $\mathrm{Yu}^{*}, \mathrm{P}-\mathrm{C}$. Yang* and the TAMI group ${ }^{\S}$

${ }^{*}$ Dept of Internal Medicine, Shuang-Ho Hospital, ${ }^{+}$Dept of Laboratory Medicine, National Taiwan University Hospital,

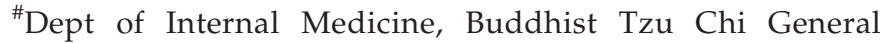
Hospital, Taipei, and "Dept of Nursing, National Taiwan University Hospital Yun-Lin Branch, Yun-Lin County, Taiwan. ${ }^{\S}$ The Taiwan Anti-Mycobacteria Investigation (TAMI) group members are as follows: J-Y. Wang, L-N. Lee, C-J. Yu, P-C. Yang, H-C. Lai, W-J. Su, C-C. Shu, C-H. Lee and M-C. Yu.

Correspondence: J-Y. Wang, Dept of Internal Medicine, National Taiwan University Hospital, \#7, Chung-Shan South Road, Taipei, 100, Taiwan. E-mail: jywang@ntu.edu.tw

Support Statement: The study was supported by the Institute for Biotechnology and Medicine, Taiwan.

Statement of Interest: None declared.

\section{REFERENCES}

1 Rieder HL. Sputum smear conversion during directly observed treatment for tuberculosis. Tuber Lung Dis 1996; 77: 124-129.

2 Catanzaro A, Horsburgh R. TBTC Study 22: risk factors for relapse with once-weekly isoniazid/rifapentine (HP) in HIV-negative TB patients. Am J Respir Crit Care Med 2000; 161: A252.

3 Tam CM, Chan SL, Kam KM, et al. Rifapentine and isoniazid in the continuation phase of treating pulmonary tuberculosis: final report. Int J Tuberc Lung Dis 2002; 6: 3-10.

4 Wang JY, Lee LN, Chou CS, et al. Performance assessment of a nested-PCR assay (the RAPID BAP-MTB) and the BD Probe Tec ET system for detection of Mycobacterium tuberculosis in clinical specimens. J Clin Microbiol 2004; 42: 4599-4603.

5 Pfyffer VV, Gutiérrez MC, Brown-Elliott B, et al. Mycobacterium. In: Murray PR, Baron EJ, Jorgensen $\mathrm{JH}$, et al., eds. Manual of Clinical Microbiology. 9th Edn. Washington, ASM Press, 2007; pp. P543-P572.

6 Diagnostic standards and classification of tuberculosis in adults and children. Am J Respir Crit Care Med 2000; 161: 1376-1395.

7 Blumberg HM, Burman WJ, Chaisson RE, et al. American Thoracic Society/Centers for Disease Control/Infectious Diseases Society of America: treatment of tuberculosis. Am J Respir Crit Care Med 2003; 167: 603-662.

8 World Health Organization. Treatment of Tuberculosis: Guidelines for National Programmes. 3rd Edn. World Health Organization, Geneva, 2003.

9 American Thoracic Society/Centers for Disease Control/Infectious Diseases Society of America: controlling tuberculosis in the United States. Am J Respir Crit Care Med 2005; 172: 1169-1227.

10 Jindani A, Aber VR, Edwards EA, et al. The early bactericidal activity of isoniazid related to its dose size in pulmonary tuberculosis. Am J Respir Crit Care Med 1997; 156: 895-890.

DOI: $10.1183 / 09031936.00102709$ 